\title{
SARS-CoV-2 Exposure from Health Care Workers to Infants: Effects and Outcomes
}

\author{
Lana A. Shaiba, MD, FRCPC ${ }^{1,2}$ Adnan Hadid ${ }^{1,2}$ Sahar H. Abdulghani, MD ${ }^{3}$ Shaikh A. Hussain ${ }^{1,4}$ \\ Prakesh S. Shah, MD, FRCPC $5,6,7$
}

\footnotetext{
${ }^{1}$ Department of Pediatrics, College of Medicine, King Saud University, Riyadh, Saudi Arabia

2 Department of Pediatrics, King Saud University Medical City, Riyadh, Saudi Arabia

3 Department of Obstetrics and Gynecology, College of Medicine, King Saud University, King Khalid University Hospital, King Saud University Medical City, Riyadh city, Saudi Arabia

${ }^{4}$ Neonatology, King Saud University Medical City, Riyadh, Saudi Arabia

${ }^{5}$ Department of Pediatrics, Mount Sinai Hospital, Toronto, Ontario, Canada

6 Department of Pediatrics, Toronto University, Toronto, Ontario, Canada

${ }^{7}$ Maternal-infant Care Research Centre, Mount Sinai Hospital,

Toronto, Ontario, Canada
}

Am J Perinatol 2023;40:799-806.
Address for correspondence Lana Shaiba, MD, FRCPC, Department of Pediatrics, College of Medicine, King Saud University, Riyadh, Saudi Arabia, King Saud University Medical City, Riyadh, KSA, Po Box 7805 Riyadh 11472, Saudi Arabia (e-mail: Ishaiba@ksu.edu.sa).

\begin{abstract}
Keywords

- SARS-CoV-2

- exposure

- health care

- workers

- infants

- effects

- outcomes

Objective This study aimed to evaluate the risk and outcomes of severe acute respiratory syndrome coronavirus 2 (SARS-CoV-2) transmission from positive health care workers (HCW) to infants in the neonatal intensive care unit (NICU) and the postnatal ward.

Study Design We conducted a retrospective analysis of infants in NICU and the postnatal ward postexposure to a COVID-19 positive HCW between May 1 and July 31, 2020. HCW had the detection of SARS-CoV-2 after being symptomatic. Infants exposed to these HCW were tested for SARS-CoV-2 and were classified as confirmed positive when test was positive 24 hours after exposure; confirmed negative when test was negative with no escalation of respiratory support provided; and probable if test was negative. However, infant required escalation of respiratory support. Infants were followed at 14 days postexposure then at the end of the study period for admitted infants.

Results A total of 31 infants were exposed to SARS-CoV-2 positive HCWs (42 exposure incidences). The median age at exposure was 21 days. None of the infants was confirmed positive. Nine infants were classified as probable cases of whom five infants with underlying chronic illnesses died, two were discharged home, and two were still admitted. Of the 22 confirmed negative cases, 15 were discharged and were well on follow-up, and 7 were still admitted.

Conclusion No active transmission of infection from infected HCW to admitted infants was identified. Although some infants had respiratory escalation postexposure none were confirmed positive. Adhering to personal protective equipment by HCW or low susceptibility of infants to SARS-CoV-2 infection may explain the lack of transmission.
\end{abstract}

received

January 14, 2021

accepted after revision

July 8, 2021

article published online

August 27, 2021
DOI https://doi.org/ 10.1055/s-0041-1735215. ISSN 0735-1631.

\footnotetext{
(C) 2021. The Author(s).

This is an open access article published by Thieme under the terms of the Creative Commons Attribution-NonDerivative-NonCommercial-License, permitting copying and reproduction so long as the original work is given appropriate credit. Contents may not be used for commercial purposes, or adapted, remixed, transformed or built upon. (https://creativecommons.org/ licenses/by-nc-nd/4.0/) Thieme Medical Publishers, Inc., 333 Seventh Avenue, 18th Floor, New York, NY 10001, USA
} 


\section{Key Points}

- There are no reported cases of transmission of SARS-CoV-2 infection from infected HCW to infants admitted to the NICU in our study.

- Adherence to personnel protective equipment is important to prevent transmission of SARS-CoV-2

- When an infant is exposed to a HCW who is positive for SARS-CoV-2 and has escalation of respiratory support, SARS-CoV2 as a cause should be investigated

Coronavirus disease 2019 (COVID-19), a pandemic declared by World Health Organization (WHO), is caused by novel coronavirus known as severe acute respiratory syndrome (SARS-COV 2). ${ }^{1-4}$ The disease is primarily an airborne infection via contact and droplet spread with respiratory tract as main portal of entry. ${ }^{5-7}$ As this disease is documented to have a strong human-to-human transmission, infants are also vulnerable. However, susceptibility and severity of disease in children is low possibly due to immature immune system or low expression of ACE2 receptors. ${ }^{8}$

The number of COVID-19 positive cases among health care workers $(\mathrm{HCW})$ in hospital settings are increasing despite of the implementation of strict infection control measures recommended by WHO. ${ }^{9}$ Around $60 \%$ of all infected people are asymptomatic or have mild symptom creating difficulty in ascertaining the disease burden on infants with possibility of transmission from infected HCW. ${ }^{10,11}$ Hospital policies have changed their measures for the prevention of SARA-CoV-2 spread during the pandemic to represent changing evidence. Measures for preventing spread of the infection include using proper personal protective equipment (PPE) by health care providers when handling and caring for infants admitted to the NICU. It is also recommended to either isolate an infant at high risk for contracting the infection postexposure to a positive $\mathrm{HCW}$ in a negative pressure room in an isolette or care for the infant in an isolette, which followed by monitoring for symptoms and assuring two nasopharyngeal aspirate swabs for SARS-CoV-2 virus are negative. ${ }^{12}$ Although transmission of infected parents and HCW is a concern previous studies have proven zero transmission of the virus from an infected HCW or parent to neonates admitted to the NICU when proper PPE is used. $^{12}$

Neonatal infection with SARS-CoV-2 is rare. A more recent metanalysis of neonatal cases of SARS-CoV-2 showed that $55 \%$ of neonates are usually asymptomatic, and the most common presenting symptom is respiratory symptoms (52\%) followed by fever then gastrointestinal symptoms. ${ }^{13}$ Infected neonates usually do well and if admitted to hospital are discharged after a median length of stay of 6 to 14 days. ${ }^{14,15}$ Our aim was to identify the risk of infection, clinical presentation, and outcome of infants in hospital settings exposed to SARS-CoV-2 viral genome positive HCW.

\section{Materials and Methods}

This was a retrospective cohort study performed at King Saud University Medical City, King Saud University Riyadh, Saudi Arabia between May 1 and July 31, 2020. The study was conducted after attaining approval from institutional review board research project number E-20-5131. All infants who came in contact with SARS-CoV-2 viral genome positive HCW during hospital stay were included in the study. The infants born to SARS-CoV-2 viral genome positive mothers and infants who were exposed to SARS-CoV-2 in community and got admitted to our hospital were excluded. The hospital formulated guidelines for all HCWs to wear proper PPE according to the area of working on April 7, 2020 to curtail the spread of infection from asymptomatic carriers within the hospital.

All HCWs in the hospital including those taking care of infants during the study period were wearing surgical mask with goggles and disposable gowns as per the hospital policy guidelines. Frequent hand washing with soap and water in between contacts and at entry and exit of unit was implemented. HCWs and the general population focused on stringent hand hygiene, and alcohol-based hand sanitizer. HCW was identified to have SARS-CoV-2 infection when tested positive by real-time fluorescence polymerase chain reaction (RT-PCR) from nasopharyngeal swab after being symptomatic. We did not have universal guidelines for testing HCW irrespective of clinical status. A HCW was advised to be tested as soon as they developed symptoms or were categorized as medium to high-risk exposure as per Saudi Centers for Disease Control and Prevention (CDC) guidelines (https:// covid19.cdc.gov.sa/). ${ }^{15}$ Once a HCW is identified as infected, they received sick leave for the duration of isolation as per Saudi CDC guidelines.

All infants who came into contact within 5 days with a HCW identified as SARS-CoV-2 positive during the study period were identified by contact tracing by our infection control department. As per the guidelines CDC, Saudi Arabia (https://covid19.cdc.gov.sa/), ${ }^{15}$ an infant was identified as a contact of a positive case who was within $2 \mathrm{~m}$ of a confirmed positive HCW case for $>15$ minutes, or infant who had direct physical contact with a confirmed COVID-19 case for their care provision. ${ }^{15}$ Infants who were considered contacts were immediately isolated in a closed incubator if their weight permits in a separate room or are managed in an open bed if they cannot be put in a closed incubator in a single room with contact and droplet precautions. All infants were cared for in a regular room unless their NP swab came back to be positive, in that case the infant is transferred to a negative pressure room. Some infants were exposed to more than one time with positive HCW.

Exposed infants were subjected to nasopharyngeal swab for the detection of viral genome of SARS-CoV-2 at $\geq 24$ hours 
and 72 hours postexposure. The test was performed by initial viral RNA extraction using Chemagic 360 Viral Nucleic acid extraction system (Perkin Elmer, Waltham, MA) and Magmax Viral Nucleic acid extraction system (Thermofisher, Waltham, MA) followed by amplification and detection using 7,500 RT-PCR (Applied Biosystems, Foster City, CA) and ViiA7 real-time fluorescence polymerase chain reaction (RT-PCR) (Applied Biosystems, Foster City, CA) on the samples collected from nasopharyngeal and oropharyngeal aspirations conducted in negative pressure rooms as per the guidelines. Blood tests were not recommended unless clinically required. The test identifies of SARS-CoV-2 E and S genes and the cycle threshold for positivity was $\leq 45$.

Exposed infants were categorized into three groups according to testing, clinical features suggestive of possible infection, and escalation in respiratory support ${ }^{16}$ :

- Confirmed cases: Detection of the SARS-CoV-2 viral genome by PCR in nasopharyngeal swab at $\geq 24$ hours after exposure.

- Probable cases: No detection of the SARS-CoV-2 viral genome by PCR in nasopharyngeal swab at $\geq 24$ hours after exposure, but there was a change of clinical status in the form of escalation of respiratory support, and gastrointestinal symptoms including vomiting and diarrhea and neurological symptoms.

- Not infected cases: No detection of the SARS-CoV-2 viral genome by PCR in nasopharyngeal swab at $\geq 24$ hours after exposure, and there was no change of clinical status in the form of escalation in respiratory support.

For the purpose of this study, we defined escalation of respiratory support as an increase in receipt of respiratory support as any of the following: (1) change from room air to needing nasal cannula oxygen or higher support; (2) increase in the $\mathrm{FiO}_{2}$ requirement by more than $5 \%$ for more than 24 hours from the baseline; (3) an increase in noninvasive respiratory support either from nasal cannula to continuous positive airway pressure (CPAP) or higher support; (4) or change from CPAP support to noninvasive positive pressure ventilation or higher support; (5) change from noninvasive positive pressure ventilation to receipt of mechanical ventilation; and (6) change from conventional ventilation to high frequency ventilation.

Follow-up information was collected on day 14 postexposure either by phone call to assess the general health condition of the infants if they were discharged home or a review of their electronic health records if the patient was still admitted in hospital for any other reasons. For infants who were considered as probable cases, the follow-up continued until death or return to baseline from a respiratory perspective.

\section{Statistical Analyses}

Descriptive statistics for numeric variables was done by using median, range, and interquartile range, while for categorical variables was done by using numbers and percentages. Bivariate analysis was done for the association between numeric variables and categorical variables by using Mann-Whitney U test and for the association between categorical variables using Chi-square test and Fisher's exact test. Statistical analysis was done by using IBM SPSS statistics, version 26 and $p$-value of $<0.05$ is considered significant.

\section{Results}

Between May 1 and July 31, 2020, a total of 31 infants were investigated after exposure to a SARS-CoV-2 positive HCW. These 31 infants had a total of 42 separate incidences of exposures ( 22 infants were exposed once, seven infants were exposed twice, and two infants were exposed three times). The mean standard deviation (SD) age of these infants at the time of exposure was 52 (9) days, and median (range) age was 21 (1-372) days. All infants were born in the hospital to SARS-CoV-2 negative mothers. Infants have had one to three postexposure swabs for SARS-CoV-2 RT-PCR tests. None of the included infants were diagnosed as confirmed SARSCoV-2 positive during any of the exposures. A total of 12 exposure incidences (in nine infants) were diagnosed as probable cases of SARS-CoV-2 infection according to postexposure escalation of respiratory support. However, 30 exposure incidences (22 infants) were confirmed negative for SARS-CoV-2 infection, as they had no change in clinical condition or needed escalation of respiratory support. The demographic characteristics of included infants (22 confirmed negative and 9 probable infections) are reported in -Table 1. Mean time of exposure in days was 1.8 (SD \pm 1.15 ) and ranged between $<1$ and 5 days. Of the confirmed negative group, 15 infants (68\%) were discharged, 7 are still admitted, and none died.

Out of 42 exposure incidences, 36 exposures were in the NICU (85.7\%) and six exposures were in the PNW (14.3\%). - Table 2 shows the descriptive statistics of the exposure incidences. After each exposure incidence, infants had at least one swab collected for SARS-CoV-2 testing. Three swabs were performed for a total of six exposures (four from confirmed negative and two from probable cases). All exposure incidences who tested negative and had no escalation of respiratory support survived $(n=30)$.

The detailed clinical characteristics of 12 exposure incidences (nine infants) classified as probable cases are given in -Table 3. Moreover, 11 out of 12 exposure incidences led to escalation of respiratory support within 5 days of exposure, and one exposure incidence led to escalation of respiratory support 9 days following exposure. None of the exposures led to gastrointestinal tract or neurological symptoms. Of nine infants in whom respiratory support was escalated, five infants (55.5\%) died prior to discharge from hospital. The cause of death in five infants who died included one case each of post patent ductus arteriosus ligation with cardiovascular instability, Severe combined immunodeficiency disease with secondary hemophagocytic lymphohistiocytosis syndrome, sepsis with leucocyte adhesion defect, respiratory failure secondary to airway malformation, and cytomegalovirus pneumonia. During the period of escalation, one infant received inotropic support, three infants had leukocytosis, and two infants developed thrombocytopenia. 


\begin{tabular}{|c|c|c|}
\hline Demographic characteristics & $\begin{array}{l}\text { Confirmed } \\
\text { negative case }\end{array}$ & $\begin{array}{l}\text { Probable case } \\
\text { of SARS-CoV-2 }\end{array}$ \\
\hline Total no. of cases (\%) & $22(70.9)$ & $9(29)$ \\
\hline Total no. of exposures (\%) & $30(71.4)$ & $12(28.5)$ \\
\hline Gestational age & $34(24-40)$ & $31(25-38)$ \\
\hline Sex (male \%) & 54.5 & 44.4 \\
\hline Number of exposures, median (range) & $2(1-3)$ & $1.5(1-2)$ \\
\hline Escalation of respiratory support post exposure & $0(0)$ & $9(100)$ \\
\hline \multicolumn{3}{|l|}{ Diagnosis at time of exposure } \\
\hline Preterm & $14(63.6)$ & $6(66.6)$ \\
\hline Chronic lung disease & $4(18.1)$ & $4(44.4)$ \\
\hline Cardiovascular condition & $5(22.7)$ & $4(44.4)$ \\
\hline Respiratory distress syndrome/transient tachypnoea of newborn & $5(22.7)$ & $1(11.1)$ \\
\hline Discharge & $22(100)$ & $4(44.4)$ \\
\hline Death & $0(0)$ & $5(55.5)$ \\
\hline
\end{tabular}

Septic work-up was performed for these five neonates. Two infants had ground glass changes on the chest X-ray, and one infant had cytomegalovirus in blood. Three infants died within 14 days postexposure and two infants died after

Table 2 Descriptive statistics of the exposure incidences to COVID-19 positive health care workers

\begin{tabular}{|c|c|c|}
\hline \multirow[t]{2}{*}{ Associated factors } & $\begin{array}{l}\text { Confirmed } \\
\text { negative } \\
\text { exposure } \\
(n=30)\end{array}$ & $\begin{array}{l}\text { Probable } \\
\text { case of } \\
\text { SARS-CoV-2 } \\
(n=12)\end{array}$ \\
\hline & $n(\%)$ & \\
\hline \multicolumn{3}{|l|}{$\begin{array}{l}\text { Age at time of } \\
\text { exposure (d) }\end{array}$} \\
\hline Median (range) & $35.5(1-570)$ & $55(3-306)$ \\
\hline \multicolumn{3}{|l|}{ Duration of exposure } \\
\hline Median (range) & $1.00(0-4)$ & $2(1-5)$ \\
\hline$<1 d$ & $2(6.6)$ & $0(0)$ \\
\hline $1 \mathrm{~d}$ & $15(50)$ & $3(25)$ \\
\hline $2 d$ & $5(16.6)$ & $5(41.6)$ \\
\hline $3 d$ & $7(23.3)$ & $2(16.6)$ \\
\hline $4 d$ & $1(3.3)$ & $1(8.3)$ \\
\hline $5 d$ & $0(0)$ & $1(8.3)$ \\
\hline \multicolumn{3}{|l|}{ Place of contact } \\
\hline $\begin{array}{l}\text { Neonatal intensive } \\
\text { care unit }\end{array}$ & $24(80)$ & $12(100)$ \\
\hline Postnatal ward & $6(20)$ & $0(0)$ \\
\hline \multicolumn{3}{|l|}{ Number of swabs } \\
\hline 1 & $30(100)$ & $12(100)$ \\
\hline 2 & $16(53.3)$ & $9(75)$ \\
\hline 3 & $4(13.3)$ & $2(16.6)$ \\
\hline
\end{tabular}

14 days postexposure. Of the four infants who survived, none received inotropic support, one had leukocytosis, and two had blood stream infection with thrombocytopenia and were treated with antibiotics. Three infants had returned to baseline with regards to respiratory support within 14 days postexposure.

\section{Discussion}

In our study, although 42 exposure incidences to SARS-CoV-2 positive HCWs happened within 5 days before confirmation of the disease, none of the exposures were confirmed to be positive in at least one nasopharyngeal swab test (some had three negative swabs). Out of these 42 exposure incidences, 30 incidences (22 infants) were classified as confirmed negative, whereas 12 incidences (in nine infants) were classified as probable case because of respiratory escalation postexposure. All infants who had no escalation of respiratory support postexposure did very well and survived, whereas five out of nine infants who had escalation of respiratory support died. The cause of death was not attributed to SARS-CoV-2 infection.

More HCWs were being infected with COVID-19 at the peak of the infection due to higher community prevalence. This led to higher exposure of neonates in our units, as they have prolonged hospitalization as well as they need prolonged contact with HCW to receive all the care they need. HCWs are at the frontline fighting against COVID-19 and possibly have significant exposure to SARS-CoV-2. The reported rate of infection among HCW range between 3.8\% in Wuhan, ${ }^{17} 6 \%$ in Netherlands, ${ }^{18}$ and $29 \%$ in a report from Hong Kong. ${ }^{19}$ The concern is that infected HCWs can transmit the disease to their patients and spread the infection. However, we did not observe such spread in our NICU as none of the infants turned out to be positive. Our findings as similar to what has been reported previously in regards to 


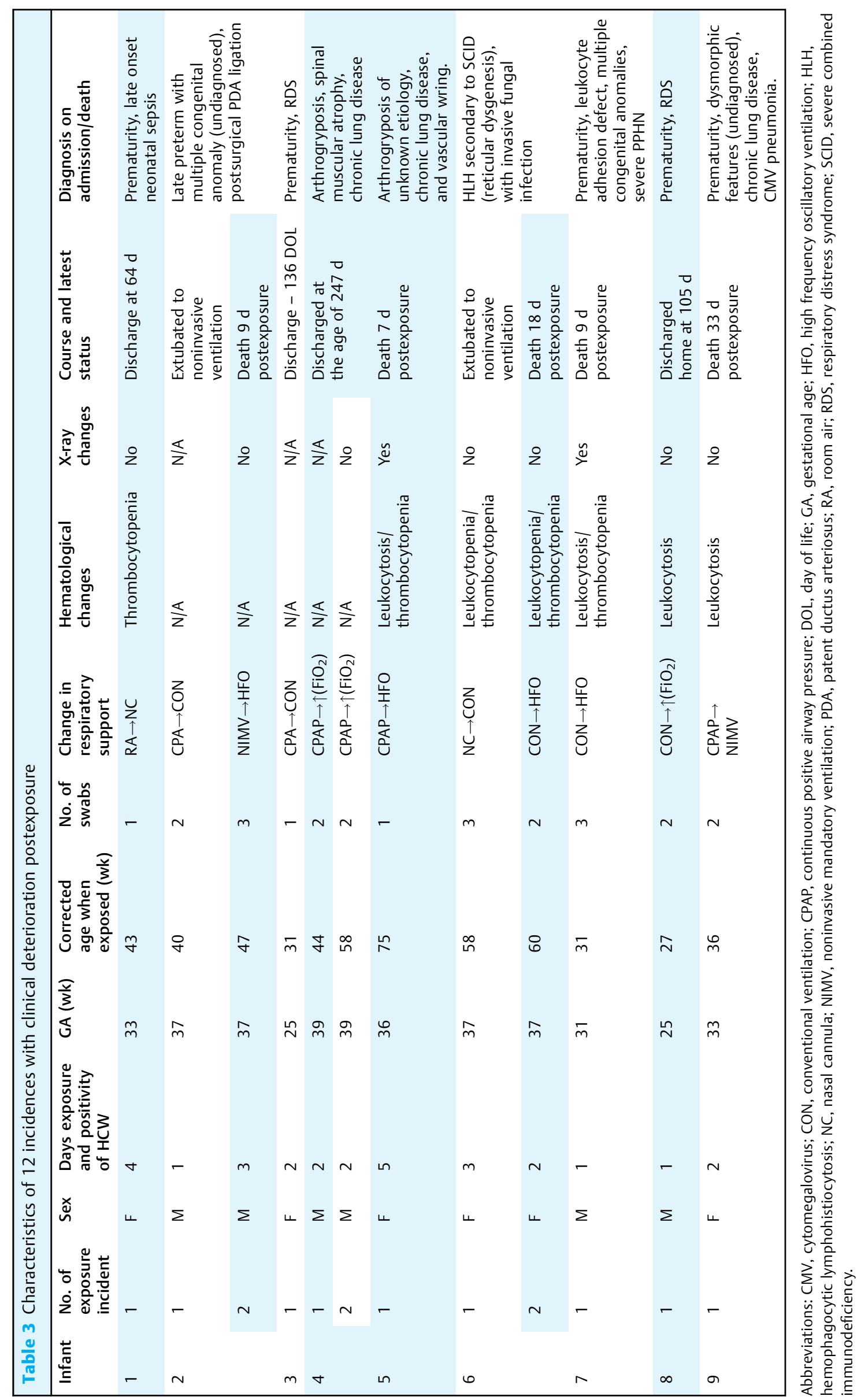


effectiveness of proper PPE use in the prevention of the spread of the virus in the NICU. ${ }^{13}$ Upon identification of SARS-CoV-2 virus as a pandemic, The Ministry of Health in collaboration with the Saudi CDC formulated and implemented hospital wide policy as stage 3 preparedness. ${ }^{15}$ The mandatory use of PPE became a standard of care. Also, department and unit specific guidelines were developed and implemented, which may have prevented the transmission of SARS-CoV-2 in our cohort. As part of the preparedness of the NICU at our hospital, nurses were expected to fill a selfadministered survey (respiratory daily log sheet for NICU staff) to advice on safety of coming to work depending on the identification of respiratory and gastrointestinal symptoms. With the spread of infection especially at the peak of the curve more HCW were being tested positive for SARS-Cov-2 infection and possibly exposing admitted patients to the virus. ${ }^{15}$ Viral transmission from infected HCW to patients continues to be a concern, especially when they are in their preclinical/pre-symptomatic period. Most studies focused on HCW protection from SARS-CoV-2 infection, and some studies had shown the efficacy of surgical face mask in decreasing SARS-CoV-2 transmission. ${ }^{20-22}$ Our study confirms these findings because despite 42 exposure incidences (including 12 exposure incidents being probable cases), none of them were confirmed as positive. As it was shown in other studies suggesting very low incidence of nosocomial SARSCoV-2 in hospitalized patients when the HCW adhered to proper PPE use. ${ }^{23}$ Surgical face masks with the other infection control measures were shown to reduce the transmission from infected mothers to their babies even with rooming-in and direct breastfeeding. ${ }^{10}$

We must acknowledge that the number of neonates exposed to HCW in our study is small so we are less certain whether no identification of confirmed positive case reflects low infectivity rate in neonates due to relative lack of ACE-2 receptors or it is due to very stringent precautions taken at our hospital using proper personal protective strategies. The recent evidence indicates that ACE2 receptor protein which play important role for entry of the virus into the host is less mature in infants and young children and thus may not function properly as a receptor for SARS-CoV-2. ${ }^{24}$ The intracellular response induced by ACE2 in the alveolar epithelial cells of infants and children is hypothesized to be lower than that of adults. This can be explained by the observation of recent data indicating that infants and children experience more SARS-CoV-2 infections in the upper respiratory tract than the lower respiratory tract. ${ }^{24}$ Systematic reviews of maternal-neonatal transmission also suggested a very low rate of transmission of infection. ${ }^{25}$ Moreover, SARS-CoV-2 virus proteins (orf1ab, ORF10, and ORF3a) in adults attack the heme on the $1-\beta$ chain of hemoglobin to dissociate the iron to form porphyrin. ${ }^{26}$ This reduces hemoglobin concentration leading to tissue hypoxia. However, in newborns and infants, the fetal hemoglobin makes up $80 \%$ of the hemoglobin, mostly made of $\alpha$ and gamma chain, which may be protective against the coronavirus. ${ }^{26}$ Systemic inflammatory response syndrome also known as cytokine storm is due to surge of activated immune cells into the lungs resulting in inflammation and fluid buildup that can lead to respiratory distress and can cause secondary bacterial pneumonia. ${ }^{27}$ This response is underdeveloped in infants and children ${ }^{27}$ and could explain the recently published CDC data, where only $54 \%$ pediatric cases had cough as compared with $80 \%$ in adults. $^{28}$ Two infants in our cohort developed respiratory symptoms and had ground glass appearance on X-ray postdeterioration. Though in neonates there are several reasons for such acute deterioration and findings, temporality of events cannot be ignored. Young immune system in this population group with its efficient T-cells may potentially perform a superior job of responding to SARS-CoV-2. ${ }^{29}$ Hence SARS-CoV-2 leaves young children and neonates with minimal or no symptoms. ${ }^{30}$ However, our findings of no confirmed transmission could be limited to settings in which children are cared for and not in other population.

The detailed description of probable cases in our series could be interpreted as potentially "no transmission" of infection to neonates. However, it also alerts clinicians to the need to remain vigilant as in many cases no other explanation for acute change was identified.

Our study has limitations including its retrospective nature with a low sample size. We attempted to identify all possible exposures in our unit. However, it is possible that we might have missed some of the exposures. Our patient population was heterogeneous ranging between neonates till infants (18 months), given the nature of our hospital policy in keeping all patients admitted to NICU under neonatal services until they are ready for discharge. Our definition of considering "possible" cases could be challenged as there could be other reasons for escalation of respiratory support especially in neonates. Indeed, we identified two cases of bacterial sepsis, which could have been reason for need for escalation in respiratory support. Given that autopsy is not culturally accepted, none of our patients underwent postmortem investigation to confirm the cause of death. The purpose of this study was not to quantify the cases of possible transmission but to identify and inform policy makers of potential outbreak mitigation strategies in health care settings. Finally, the report is coming from a single center and further validation using stricter criteria are needed.

Our study highlights importance of proper use of PPE in preventing spread of infection. Though none of infants became truly infected postexposure in our case series, some infants worsened from their baseline status. Further reports from other settings, where HCWs are routinely screened by viral genome testing will help delineate the nature, extent, and magnitude of this issue especially in high acute care settings of newborn intensive care units.

In conclusion, in this case series of infant's clinical followup post-SARS-CoV-2 exposure from HCW, we identified no active transmission of infection from HCW to infants. We noted clinical deterioration in some infants post exposure. However, none of them were confirmed to be positive cases. This lack of transmission could be attributed to stricter use of PPE for all close patient encounters or low susceptibility of infants to SARS-CoV-2 infection. However, further studies are warranted. 


\section{Conclusion}

We identified the risk of infection, clinical presentation, and outcome of infants exposed to infected HCWs.

Note

This study is under the registry's name, (King Saud University Medical City) registration number (20/0613/ IRB) (E-20-5130), and data sharing statement (all available data can be obtained by contacting the corresponding author).

De-identified individual participant data (including data dictionaries) will be made available, in addition to study protocols, the statistical analysis plan, and the informed consent form. The data will be made available upon publication to researchers who provide a methodologically sound proposal for use in achieving the goals of the approved proposal. Proposals should be submitted to (lshaiba@ksu.edu.sa),00966-50-5480202.

\section{Authors' Contributions}

L.A.S. conceptualized and designed the study, drafted the initial manuscript, and reviewed and revised the manuscript. A.A.H. and S.A.H. designed the data collection instruments, collected data, performed the initial analyses, and reviewed and revised the manuscript. S.A.H. and P.S.S. conceptualized and designed the study, coordinated and supervised data collection, and critically reviewed the manuscript for important intellectual content. All authors approved the final manuscript as submitted and agree to be accountable for all aspects of the work.

Funding

None.

Conflict of Interest

None declared.

\section{References}

1 Zhu N, Zhang D, Wang W, et al; China Novel Coronavirus Investigating and Research Team. A novel coronavirus from patients with pneumonia in China, 2019. N Engl J Med 2020;382(08): 727-733

2 Huang C, Wang Y, Li X, et al. Clinical features of patients infected with 2019 novel coronavirus in Wuhan, China. Lancet 2020;395 (10223):497-506

3 Chen N, Zhou M, Dong X, et al. Epidemiological and clinical characteristics of 99 cases of 2019 novel coronavirus pneumonia in Wuhan, China: a descriptive study. Lancet 2020;395(10223): 507-513

4 Kakodkar P, Kaka N, Baig MN. A comprehensive literature review on the clinical presentation, and management of the pandemic coronavirus disease 2019 (COVID-19). Cureus 2020;12(04):e7560

5 Hoffmann M, Kleine-Weber H, Schroeder S, et al. SARS-CoV-2 cell entry depends on ACE2 and TMPRSS2 and is blocked by a clinically proven protease inhibitor. Cell 2020;181(02):271-280.e8

6 Zhou P, Yang XL, Wang XG, et al. A pneumonia outbreak associated with a new coronavirus of probable bat origin. Nature 2020;579 (7798):270-273

7 Diaz JH. Hypothesis: angiotensin-converting enzyme inhibitors and angiotensin receptor blockers may increase the risk of severe
COVID-19. J Travel Med 2020 May 1827(03):taaa041. PMID: 32186711; PMCID: PMC7184445

8 Wiersinga WJ, Rhodes A, Cheng AC, Peacock SJ, Prescott HC. Pathophysiology, transmission, diagnosis, and treatment of coronavirus disease 2019 (COVID-19): a review. JAMA 2020;324(08): 782-793

9 Lai X, Wang M, Qin C, et al. Coronavirus disease 2019 (COVID2019) infection among health care workers and implications for prevention measures in a tertiary hospital in Wuhan, China. JAMA Netw Open 2020;3(05):e209666

10 Chao JY, Derespina KR, Herold BC, et al. Clinical characteristics and outcomes of hospitalized and critically ill children and adolescents with coronavirus disease 2019 at a tertiary care medical center in New York City. J Pediatr 2020;223:14-19.e2

11 Wang J, Pan L, Tang S, Ji JS, Shi X. Mask use during COVID-19: a risk adjusted strategy. Environ Pollut. 2020 Nov;266(Pt 1):115099. Epub 2020 Jun 25. PMID: 32623270; PMCID: PMC7314683.Home. (2020, March 16). Accessed November 9, 2020 at: https://covid19. cdc.gov.sa/

12 Chandrasekharan P, Vento M, Trevisanuto D, et al. Neonatal resuscitation and postresuscitation care of infants born to mothers with suspected or confirmed SARS-CoV-2 infection. Am J Perinatol 2020;37(08):813-824

13 Cavicchiolo ME, Trevisanuto D, Lolli E, et al. Universal screening of high-risk neonates, parents, and staff at a neonatal intensive care unit during the SARS-CoV-2 pandemic. Eur J Pediatr 2020;179 (12):1949-1955

14 Raschetti R, Vivanti AJ, Vauloup-Fellous C, Loi B, Benachi A, De Luca D. Synthesis and systematic review of reported neonatal SARS-CoV-2 infections. Nat Commun. 2020;11(01):5164 11d

15 Pathak EB, Salemi JL, Sobers N, Menard J, Hambleton IR. COVID-19 in children in the United States: intensive care admissions, estimated total infected, and projected numbers of severe pediatric cases in 2020. J Public Health Manag Pract 2020;26(04):325-333

16 Shah PS, Diambomba Y, Acharya G, Morris SK, Bitnun A. Classification system and case definition for SARS-CoV-2 infection in pregnant women, fetuses, and neonates. Acta Obstet Gynecol Scand 2020;99(05):565-568

$17 \mathrm{Wu} Z$, McGoogan JM. Characteristics of and important lessons from the coronavirus disease 2019 (COVID-19) outbreak in China: summary of a report of 72314 cases from the Chinese Center for Disease Control and Prevention. JAMA 2020;323(13):1239-1242

18 Kluytmans-van den Bergh MFQ Buiting AGM, Pas SD, et al. Prevalence and clinical presentation of health care workers with symptoms of coronavirus disease 2019 in 2 Dutch hospitals during an early phase of the pandemic. JAMA Netw Open 2020;3 (05):e209673

19 Wong SCY, Kwong RT, Wu TC, et al. Risk of nosocomial transmission of coronavirus disease 2019: an experience in a general ward setting in Hong Kong. J Hosp Infect 2020;105(02):119-127

20 Sommerstein R, Fux CA, Vuichard-Gysin D, et al; Swissnoso. Risk of SARS-CoV-2 transmission by aerosols, the rational use of masks, and protection of healthcare workers from COVID-19. Antimicrob Resist Infect Control 2020;9(01):100

21 Chu DK, Akl EA, Duda S, Solo K, Yaacoub S, Schünemann HJCOVID-19 Systematic Urgent Review Group Effort (SURGE) study authors. Physical distancing, face masks, and eye protection to prevent person-to-person transmission of SARS-CoV-2 and COVID-19: a systematic review and meta-analysis. Lancet 2020;395(10242): 1973-1987

22 Kaur R, Weiss TT, Perez A, et al. Practical strategies to reduce nosocomial transmission to healthcare professionals providing respiratory care to patients with COVID-19. Crit Care 2020;24 (01):571

23 Rhee C, Baker M, Vaidya V, et al; CDC Prevention Epicenters Program. Incidence of nosocomial COVID-19 in patients hospitalized at a large US Academic Medical Center. JAMA Netw Open 2020;3(09):e2020498 
Shaiba et al.

24 Cruz AT, Zeichner SL. COVID-19 in children: initial characterization of the pediatric disease. Pediatrics 2020;145(06):e20200834

25 Walker KF, O’Donoghue K, Grace N, et al. Maternal transmission of SARS-COV-2 to the neonate, and possible routes for such transmission: a systematic review and critical analysis. BJOG 2020;127 (11):1324-1336

26 Wong P, Weerakul J, Sritippayawan S. Hemoglobin analysis in the first year of life. Mediterr J Hematol Infect Dis 2016;8(01):e2016012

27 Jeljeli M, Guérin-El Khourouj V, Pédron B, Gressens P, Sibony O, Sterkers G. Ontogeny of cytokine responses to PHA from birth to adulthood. Pediatr Res 2019;86(01):63-70
28 CDC COVID-19 Response Team. Coronavirus disease 2019 in children - United States, February 12-April 2, 2020. MMWR Morb Mortal Wkly Rep 2020;69(14):422-426

29 Shearer WT, Rosenblatt HM, Gelman RS, et al; Pediatric AIDS Clinical Trials Group. Lymphocyte subsets in healthy children from birth through 18 years of age: the Pediatric AIDS Clinical Trials Group P1009 study. J Allergy Clin Immunol 2003;112(05): 973-980

30 Rawat M, Chandrasekharan P, Hicar MD, Lakshminrusimha S. COVID-19 in newborns and infants-low risk of severe disease: silver lining or dark cloud? Am J Perinatol 2020;37(08):845-849 